\title{
QUELQUES ASPECTS ANCIENS ET MODERNES DE LA PHOTOSYNTHẼSE
}

\author{
par J.-M. BOVE \\ Institut Français de Recherches Fruitières Outre-Mer (I.F.A.C.). \\ (Service de biochimie).
}

\begin{abstract}
Des progrès considérables ont été réalisés dans certains domaines de la Photosynthèse au cours des toutes dernières années. Cés progrès ont non seulement permis d'éclaircir le mécanisme de certaines réactions de la Photosynthèse, mais encore ils nous obligent à modifier la conception même que nous avions de cette fonction physiologique capitale. Cela est si vrai qu'on hésiterait maintenant à définir la Photosynthèse comme la biosynthèse de substances carbonées au moyen de l'énergie lumineuse à partir du gaz carbonique de l'air!

L'un des buts de cet article sera de montrer que la Photosynthèse, au sens strict du mot, est davantage une assimilation de phosphate inorganique que de gaz carbonique. Nous nous attacherons moins au mécanisme détaillé des diverses réactions qu'à la place qu'elles occupent dans le concept actuel de la Photosynthèse.
\end{abstract}

PREMIERE PARTIE

\section{LA PHOtosynthéSE de PRIESTLey A VAN NIEL}

\section{Historique.}

Ce n'est qu'à une époque relativement récente, le $\mathrm{xvII}^{\mathrm{e}}$ siècle, que la photosynthèse a été découverte. Des premières observations de Priestley, en I77I, jusqu'au livre de Mayer, en I845, où l'accent est mis sur l'énergie lumineuse en tant que source de toute énergie dans les plantes, trois quarts de siècle se sont écoulés. La photosynthèse eut une naissance difficile.

Jusqu'au $\mathrm{xvII}^{\mathrm{e}}$ siècle, il était communément admis, à la suite d'Aristote et de Théophraste, que les plantes se nourrissaient exclusivement de l'«humus » et de l'eau du sol. Personne n'aurait songé à faire intervenir l'oxygène, le gaz carbonique et l'énergie lumineuse dans la nutrition des plantes. Il faudra attendre I840 pour voir la théorie de l'" humus » définitivement taillée en pièces sous les coups de Liebig.

$\mathrm{Au} \mathrm{XVIII}^{\mathrm{e}}$ siècle, non seulement la théorie de l'humus était très généralement acceptée, mais les noms même de gaz carbonique, d'oxygène, ..., étaient inconnus. On était en pleine théorie phlogistique. $\mathrm{Ce}$ n'est qu'après la publication des travaux de Lavoisier, en 1785 , que la terminologie moderne commença à se répandre, et qu'on parla d'oxygène au lieu d'air déphlogistiqué, et de gaz carbonique à la place de l'air phlogistiqué ou de l'air "fixe".

\section{a) Joseph Priestley (I733-I804) : La purification de l'air par les plantes.}

J. Priestley, théologien, chimiste et physicien anglais, découvrit que les plantes ont le pouvoir de "purifier» l'air vicié. Voici comment il rend compte de sa découverte :

"Le I7 août I77I, je plaçai un rejet de menthe dans une certaine quantité d'air dans lequel une bougie s'était éteinte et je trouvai que le 27 du même mois une autre bougie y brûlait parfaitement bien. » (*) (Priestley, I772.) Ailleurs, il écrit : "Cette obser-

(*) Toutes les citations contenues dans cet article sont traduites à partir du Texte original. 
vation m'amena à conclure que les plantes, au lieu d'affecter l'air de la même manière que la respiration animale, inversent les effets de la respiration et tendent à maintenir l'atmosphère douce et saine, quand elle est devenue nuisible à cause d'animaux qui y vivent et $\mathrm{y}$ respirent ou qui $\mathrm{y}$ meurent et $\mathrm{y}$ pourrissent. " (Priestley, I776.)

Priestley a donc bien vu que les plantes avaient une influence sur l'atmosphère dans laquelle elles se développaient, mais il ne s'est absolument pas rendu compte que la lumière était nécessaire. Ce n'est que par le plus grand des hasards que ses plantes en expérimentation recevaient suffisamment de lumière. C'est à Ingen-Housz que revient le mérite d'avoir compris l'importance de la lumière.

\section{b) Jan Ingen-Housz (I730-I799) : L'importance de la lumière.}

J. Ingen-Housz, médecin hollandais à la cour d'Autriche, connaissait les travaux de Priestley. A la notion que les plantes purifiaient l'air, il ajouta celle de l'importance de la lumière. En I779, il écrit : "cette opération merveilleuse - [la purification de l'air par les plantes] - n'est aucunement due à la croissance de la plante, mais à l'influence de la lumière sur la plante. ” (Ingen-Housz, I779.)

De plus, il montre que seules les parties vertes des plantes ont le pouvoir de purifier l'air alors que les fleurs, les racines et les fruits non verts produisent de l'air vicié. Il contribue ainsi à montrer que la production d'air vicié [respiration] n'est pas l'apanage des animaux. Finalement, en utilisant des feuilles immergées dans l'eau, il montre que ces feuilles donnent lieu à une production effective d'air "déphlogistiqué " : les bulles viennent éclater à la surface de l'eau.

\section{c) Jean Senebier (I742-I809) et Jan Ingen-Housz : Le " gaz carbonique", nourriture de la plante.}

J. Senebier, théologien et bibliothécaire suisse, prolongea l'œuvre de Priestley et de Ingen-Housz en affirmant que l'“air fixe" (le gaz carbonique) est la nourriture que les plantes tirent de l'air qui les baigne ». (Senebier, I782-I783.) Cette idée fut exprimée en termes modernes en 1796 par-Ingen-Housz qui, après que Lavoisier eût mis en évidence la composition du gaz carbonique, déduisit que cette substance était une source de carbone pour la plante. La fin du xvmi ${ }^{\mathrm{e}}$ siècle fut marquée par d'assez vives polémiques entre Senebier et Ingen-Housz sur la priorité de la découverte de l'absorption du gaz carbonique. Certains historiens n'ont pas hésité à attribuer le mérite de cette découverte presque exclusivement à Ingen-Housz (Hansen I882). Il serait intéressant de reprendre ce point d'Histoire et de confronter année par année les publications de ces deux auteurs.

\section{d) Nicolas Théodore de Saussure (1767-1845): La rigueur scientifique.}

Le début du xix ${ }^{e}$ siècle coïncide avec le début de la chimie moderne. Lavoisier vient d'élucider la composition chimique du gaz carbonique. Cavendish a réalisé la synthèse de l'eau à partir de l'hydrogène et de l'oxygène.

Dans ce nouveau climat scientifique, de Saussure fut le premier, en Physiologie Végétale, à tirer parti des notions nouvelles. Avec lui, l'expérimentation devient rigoureusement quantitative. Il ne tire de conclusions qu'à partir de chiffres rigoureusement contrôlés. Ses résultats sont parmi les premiers à être exprimés en termes modernes.

Il publie ses "Recherches Chimiques sur la Végétation" en 1804 et précise, dès le début : "Les fonctions de l'eau et des gaz dans la nutrition des végétaux, les changements qu'ils font subir à leur atmosphère, sont les sujets que j'ai le plus approfondis. Les observations de Priestley, de Senebier, d'IngenHousz, ont ouvert la carrière que $j$ 'ai parcourue ; mais elles n'ont point atteint le but que je me suis proposé. " (De Saussure, I804.)

En répétant les expériences de ses prédécesseurs, avec une exactitude beaucoup plus grande, il montre que le gaz carbonique à haute concentration est toxique pour la plante, mais que des concentrations beaucoup plus élevées que celle de l'air normal ont une action bénéfique sur la croissance de la plante.

Il montre également que le dégagement d'oxygène dépend de la présence de $\mathrm{CO}_{2}$ et que la quantité $\mathrm{d}^{\prime} \mathrm{O}_{2}$ dégagé est proportionnelle à la quantité de $\mathrm{CO}_{2}$ initialement présent. Ces résultats, qui sont exacts, l'amènent à écrire : "les plantes...n'exhalent du gaz oxygène que par la décomposition immédiate du gaz acide carbonique » (de Saussure, I804), ce qui s'est révélé ne pas être exact : l'oxygène dégagé ne provient pas de $\mathrm{CO}_{2}$ mais de l'eau, ainsi que l'ont montré définitivement Ruben et coll. en ... I94I (voir plus loin).

Bien que de Saussure n'ait pas soupçonné l'oxygène de l'eau comme source de l'oxygène émis pendant la photosynthèse, l'une de ses contributions les plus importantes porte néanmoins sur le rôle de l'eau dans 
la nutrition de la plante : "On ne saurait douter que la plus grande partie de l'hydrogène que les plantes acquièrent en se développant à l'air libre, à l'aide de l'eau distillée, n'ait son origine dans ce liquide... » (de Saussure, I804).

$$
\begin{aligned}
& \text { e) Julius Robert Mayer (I8I4-1878) : } \\
& \text { Les végétaux transformateurs d'énergie solaire } \\
& \text { en énergie chimique. }
\end{aligned}
$$

En I842, Mayer, physicien et médecin allemand, établit pour la première fois l'équivalence entre l'énergie thermique et l'énergie mécanique. Il énonce la loi de la conservation de l'énergie sous le nom de Loi des Sommes de Chaleur Constante. En I845, il applique ces lois aux systèmes biologiques et précise, en un langage imagé, que l'énergie utilisée par les plantes pour leur métabolisme provient de l'énergie de la lumière solaire, l'énergie lumineuse est transformée par les plantes en énergie chimique :

"La Nature s'est donné la tâche de capter au vol la lumière déversée sur le monde et d'emmagasiner la plus mouvante de toutes les forces, après l'avoir immobilisée. Pour y parvenir, elle a recouvert l'écorce terrestre d'organismes qui, à l'état vivant, absorbent la lumière solaire et produisent, continuellement, en utilisant cette force, une somme de différence chimique. Ces organismes sont les plantes... Dans les plantes, il ne se produit qu'une transformation, non pas une création de matière..." (Mayer, I845.)

Ingen-Housz avait, le premier, mis l'accent sur la nécessité de la lumière. Mayer compléta cette notion, en formulant clairement que la lumière intervenait par son énergie.

Ainsi ce n'est que vers le milieu du XIx ${ }^{\mathrm{e}}$ siècle qu'a été reconnu le rôle capital que jouent les plantes sur notre planète.

En l'espace de 74 ans, entre la découverte de Priestley en I77I et l'énoncé des lois de Mayer, en I845, les plantes ont été promues du rôle d'agents purificateurs de l'air vicié au rôle de transformateur et d'emmagasineur de l'énergie solaire.

$\mathrm{Si}$, en $\mathrm{I} 850$, les grandes lignes de ce processus pour lequel Bornes proposera le nom de photosynthèse en I 898 seulement, sont dorénavant établies, il fallut néanmoins toute l'ardeur de Justus Liebig pour faire admettre au cours de la deuxième moitié du xIx ${ }^{\mathrm{e}}$ siècle, que les plantes éclairées absorbent et assimilent le gaz carbonique en donnant lieu à une production concomittante d'oxygène, le tout grâce à l'énergie lumineuse.

\section{Le chloroplaste, siège de l'émission d'oxygène.}

Ingen-Housz avait montré que, seules, les parties vertes des plantes avaient le pouvoir de purifier l'air.

Pelletier et Caventou, en $\mathrm{I} 8 \mathrm{I} 8$, proposèrent le nom de chlorophylle pour cette matière verte contenue dans les feuilles.

Les chloroplastes, siège de la chlorophylle, furent décrits pour la première fois, par H. v. Mohl, en 1837 ; Mohl observa également des grains d'amidon dans ces chloroplastes (Mohl, I837).

C'est cependant à Julius Sachs que revient le mérite d'avoir pleinement entrevu les relations entre la chlorophylle, les chloroplastes, la photosynthèse et l'amidon. Il est vrai que Dutrochet avait déjà insisté sur la nécessité de la chlorophylle pour la photosynthèse, (Dutrochet, I837) alors que de Saussure, induit en erreur par l'observation que de nombreuses feuilles étaient rouges, avait mis en doute l'indispensabilité de la chlorophylle, verte.

Sachs, à la suite de ses expériences sur la formation d'amidon à la lumière, est persuadé que c'est le chloroplaste lui-même qui décompose le gaz carbonique et assimile la substance organique. En faveur de cette idée, le premier argument de Sachs est le fait que : "les cellules ne se mettent pas à assimilier aussi longtemps qu'elles n'ont pas de chlorophylle verte. " (Sachs, I887.)

Mais son argument principal est le fait que : "le premier produit visible de l'assimilation (l'amidon) n'apparaît pas dans un endroit quelconque de la cellule, mais dans le chloroplaste lui-même." (Sachs, I887.) A ceux qui n'étaient pas convaincus, et qui considéraient la cellule entière, et non le seul chloroplaste, comme responsable de l'assimilation, Sachs répliquait que cela revenait "quelque peu à dire que ce n'est pas l'œil qui est l'organe de la vue, car quand on le sort de la tête, il n'a plus la possibilité de voir. " (Sachs, I887.)

Les conceptions de Sachs devaient, peu après, recevoir une confirmation directe : Engelmann, en I88I, réussit à isoler des chloroplastes de diverses plantes et à démontrer, pour la première fois, un dégagement d'oxygène en dehors de la cellule entière par ces chloroplastes illuminés (Engelmann, I882).

Engelmann avait développé une technique nouvelle, très fine, pour déceler des traces d'oxygène. Cette technique, basée sur la motilité de certaines bactéries chimiotactiques en présence d'oxygène, sera toujours citée comme un exemple d'élégance expérimentale. La méthode consiste à observer sous le microscope une suspension de chloroplastes en présence 
de ces bactéries. En l'absence d'oxygène, les bactéries sont immobiles; dès qu'on éclaire la préparation, les bactéries se mettent en mouvement et se rassemblent autour des chloroplastes, sources d'oxygène.

C'est au moyen de la même technique qu'Engelmann étudia l'action des diverses régions du spectre lumineux sur la photosynthèse. L'expérience consiste à éclairer au moyen d'un microspectre de prisme un filament d'algue et à noter, à travers le microscope, la distribution des bactéries le long du filament (fig. I).

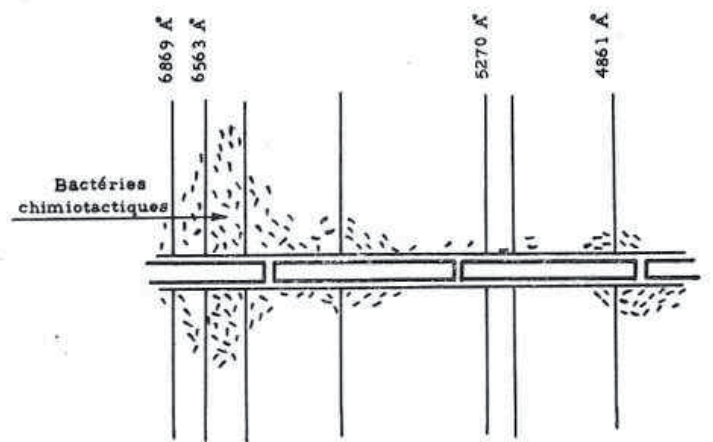

FIG. I. - Fragment de Cladophora, algue filamenteuse, dont la celulle contient de nombreux chloroplastes uniformément répartis.

Le filament est éclairé par un microspectre de lumière solaire; les bactéries chimiotactiques, sensibles à l'oxygène, se rassemblent daus les régions du spectre qui induisent la plus grande émission d'oxygène, donc qui sont les plus efficaces.

Le système optique pour la production du microspectre fut construit par Carl Zeiss. C'est là un bon exemple de coopération entre le biologiste et le constructeur d'appareil.

Enfin un mérite non moins important d'Engelmann est d'avoir reconnu le premier que certaines bactéries, les rhodo-bactéries entre autres, étaient des organismes photosynthétiques. Nous verrons plus loin combien l'étude des bactéries photosynthétiques a fait progresser la photosynthèse.

Quelques années plus tard, Beijerinck simplifiera la technique d'Engelmann en remplaçant les bactéries chimiotactiques par des bactéries qui deviennent lumineuses en présence d'oxygène. Le microscope devenait inutile, puisque la luminescence était visible à l’œil nu.

\section{Les idées de Pfeffer et de Molish : 1e chloro- plaste siège de la photosynthèse ?}

La conclusion des travaux précédents, c'est Pfeffer qui l'énonce, peu avant I9oo : «L'assimilation même du $\mathrm{CO}_{2}$ a lieu probablement entièrement dans le chloroplaste car, au moyen de la méthode bactérienne si fine, on peut montrer que des chloroplastes isolés continuent de dégager de l'oxygène à la lumière... quand ils sont placés dans une solution de sucre isoosmotique." (Pfeffer, Igoo.)

28 années plus tard, en I925, Molish, à la suite de nombreuses expériences pour lesquelles il utilisait toujours encore la technique des bactéries lumineuses de Beijerinck, fera siennes les conclusions de Pfeffer et considérera que la photosynthèse peut être effectuée par le seul chloroplaste, en dehors de la cellule vivante. (Molish, I925.)

L'idée que l'intégrité de la cellule entière ne fut pas une condition sine qua non pour la réalisation de la photosynthèse n'avait rien de choquant au début de ce siècle. En effet, Büchner venait de montrer qu'un extrait de levure, débarrassé de toutes cellules entières, avait la capacité de faire fermenter le sucre. (Büchner, I897.) Cette découverte sensationnelle, qui arrivait deux ans après la mort de Pasteur, clôturait les âpres disputes entre partisans de Pasteur, pour lesquels il n'y avait pas de fermentation sans cellule bactérienne vivante et ceux de Liebig, pour lesquels des "ferments" ou "diastases", doués de pouvoir catalytique, étaient capables en l'absence de tout organisme vivant, de faire fermenter.

Ainsi donc, si la fermentation ne nécessitait pas la participation de la cellule vivante, rien n'empêchait de penser que la photosynthèse pouvait, elle aussi, opérer en dehors de la cellule vivante, et dans le seul chloroplaste.

Nous avons vu que, pour Pfeffer et Molish, le chloroplaste était le lieu où s'accomplissait la photosynthèse parce qu'il émettait de l'oxygène quand il était éclairé. Ce dégagement d'oxygène était considéré comme un critère nécessaire et suffisant de la photosynthèse. En effet, on admettait très généralement à l'époque que l'oxygène émis provenait de la photodécomposition du $\mathrm{CO}_{2}$ absorbé. Dans ces conditions, la mesure de l'oxygène émis était ipso facto un test et une mesure du $\mathrm{CO}_{2}$ absorbé.

Mais cette conception était critiquable et les arguments à son encontre étaient les suivants :

I) Bien qu'il fût indubitable que les chloroplastes illuminés émissent de l'oxygène, la quantité d'oxygène ainsi dégagé était infime et il fallait le secours d'une technique ultra-sensible pour le détecter. (bactéries sensibles à l'oxygène.)

2) Des mesures quantitatives de l'oxygène émis n'étaient pas possibles. 
3) Il n'y avait aucune évidence expérimentale d'une fixation simultanée de $\mathrm{CO}_{2}$.

\section{Van Niel : La photolyse de l'eau.}

C'est l'étude comparée de la photosynthèse chez les bactéries photosynthétiques et les plantes vertes qui permit à Van Niel (*) de formuler de nouvelles hypothèses qui se sont révélées extrêmement fructueuses. Elles ont permis de clarifier le domaine de la photosynthèse, d'imaginer et d'effectuer un grand nombre d'expériences qui ont donné un essor considérable à la photosynthèse au cours des trente dernières années.

Donnons la parole à (Van Niel, I929) : “Qu'est ce que la photosynthèse ? Depuis longtemps on sait que, pendant la photosynthèse, une réaction a lieu qu'on peut représenter schématiquement par la formule :

$$
\mathrm{CO}_{2}+\mathrm{H}_{2} \mathrm{O}=\left(\mathrm{CH}_{2} \mathrm{O}\right)+\mathrm{O}_{2}
$$

Donc nous pouvons dire qu'elle représente un processus d'oxydo-réduction typique. $\mathrm{CO}_{2}$ est réduit, $\mathrm{H}_{2} \mathrm{O}$ est oxydé. C'est Thunberg qui, le premier, a appliqué à ce processus la théorie des phénomènes d'oxydo-réduction, développée par $\mathrm{H}$. Wieland. D'après le concept de Wieland, toutes les réactions d'oxydation impliquent le transport d'hydrogène d'une molécule, le donneur, à une autre molécule, l'accepteur. Thunberg a fait remarquer que la réduction du $\mathrm{CO}_{2}$ pendant la photosynthèse s'effectuait d'après le schéma général de Wieland; en d'autres mots : que $\mathrm{CO}_{2}$ jouait le rôle d'accepteur d'hydrogène, $\mathrm{H}_{2} \mathrm{O}$ celui de donneur et que, en conséquence, l'oxygène dégagé devait être considéré comme de $\mathrm{l}^{\prime} \mathrm{H}_{2} \mathrm{O}$ déshydrogénée :

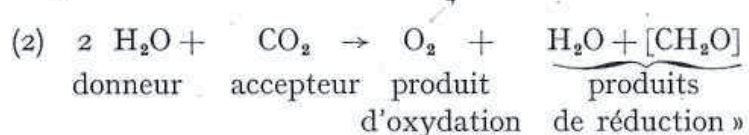

Comme cette citation le laisse entendre, la source de l'oxygène dégagé au cours de la photosynthèse ne serait pas $\mathrm{CO}_{2}$ mais $\mathrm{H}_{2} \mathrm{O}$ : l'oxygène dégagé est de l'eau déshydrogénée. Van Niel fait remarquer que la décomposition chimique de l'eau avec dégagement concomitant d'oxygène est une réaction qui nécessite de l'énergie (Van Niel, I94I, p. 322) :

$$
\text { (3) } 2 \mathrm{H}_{2} \mathrm{O} \rightarrow \mathrm{O}_{2}+4 \mathrm{H}^{+}+4^{\mathrm{e}}
$$

(*) Maintenant Professeur de Biologie a Hopkins Marine Station, Pacific Grove, Californie. ou

(4)

$$
{ }_{4} \mathrm{OH}^{-} \rightarrow \mathrm{O}_{2}+2 \mathrm{H}_{2} \mathrm{O}+4^{\mathrm{e}}
$$

$e=$ électron.

Une telle réaction se produit à l'anode d'une cuve à électrolyse remplie par exemple d'une solution alcaline diluée à travers laquelle on fait passer un courant. Les électrons passent dans l'anode et l'oxygène se dégage. Pour que cette réaction ait lieu, il faut que la différence de potentiel appliquée aux bornes des électrodes soit supérieure à une valeur minimum qui est de l'ordre de $\mathrm{I}, 7$ volt à $20^{\circ}$ pour des solutions molaires de bases. (Audubert, I953.)

$\mathrm{Si}$ on fait passer à travers la solution électrolytique une quantité d'électricité égale à I Faraday ou 96500 Coulombs, l'énergie minimum qui aura été fournie pour produire la réaction (4) est, d'après la formule : Volt $\times$ Coulombs $=$ Joules :

$$
\mathrm{I}, 7 \times 96550=164050 \text { joules }
$$

ou

$$
\frac{\mathrm{I} 64 \cdot 050}{4, \mathrm{I} 85 \times 10^{3}} \mathrm{~K} \mathrm{cal}=39,2 \mathrm{~K} \mathrm{cal}
$$

Dans la photosynthèse, c'est la lumière qui fournit l'énergie nécessaire; la première hypothèse de Van Niel consiste à écrire :

$$
\mathrm{H}_{2} \mathrm{O} \stackrel{\text { lumière }}{\longrightarrow}(\mathrm{H})+(\mathrm{OH})
$$

Autrement dit, la lumière réalise, avec l'aide de la chlorophylle et d'une série d'enzymes, une "photolyse ", une "photo décomposition" de l'eau, avec production, en quantités stœchiométriques, d'un système réducteur $(\mathrm{H})$ et d'un système oxydant $(\mathrm{OH})$. Van Niel fait bien remarquer que $(\mathrm{H})$ ne doit pas être considéré comme un atome d'hydrogène, ni $(\mathrm{OH})$ comme un radical hydroxyl. Dans son esprit, la réaction (5) «ne représente que la quintessence de la réaction photochimique ». (Van Niel, I94I, p. 323.)

Dans l'hypothèse de Van Niel, le sort de $(\mathrm{H})$ et de $(\mathrm{OH})$ va être le suivant :

I) Le système réducteur $(\mathrm{H})$ va fournir l'hydrogène qui aboutira au gaz carbonique et le réduira :

$$
4(\mathrm{H})+\mathrm{CO}_{2} \rightarrow\left(\mathrm{CH}_{2} \mathrm{O}\right)+\mathrm{H}_{2} \mathrm{O}
$$

$\left(\mathrm{CH}_{2} \mathrm{O}\right)$ représente un produit de réduction du gaz carbonique. Dans l'esprit de Van Niel, ce n'est pas nécessairement le gaz carbonique lui-même qui est réduit. $\mathrm{CO}_{2}$, avant d'être réduit, pourrait servir à carboxyler un composé organique. C'est le produit 
de cette carboxylation qui pourrait être réduit. Nous verrons plus tard combien cette vue était exacte.

2) Le système oxydant $(\mathrm{OH})$ formera une sorte de peroxyde qui, ensuite, perdra de l'oxygène :

(7) $4(\mathrm{OH}) \stackrel{\text { lumière }}{\longrightarrow}$ "peroxyde $\rightarrow 2 \mathrm{H}_{2} \mathrm{O}+\mathrm{O}_{2}$

En résumé,

(5) $\times 4{ }_{4} \mathrm{H}_{2} \mathrm{O} \rightarrow 4(\mathrm{H})+4(\mathrm{OH})$

$$
4(\mathrm{H})+\mathrm{CO}_{2} \rightarrow\left(\mathrm{CH}_{2} \mathrm{O}\right)+\mathrm{H}_{2} \mathrm{O}
$$

(7)

$$
\begin{aligned}
& \frac{4(\mathrm{OH}) \rightarrow}{2 \mathrm{H}_{2} \mathrm{O}+\mathrm{CO}_{2} \rightarrow \mathrm{H}_{2} \mathrm{O}+\mathrm{O}_{2}} \\
& \rightarrow \mathrm{O}_{2}+\mathrm{H}_{2} \mathrm{O}+\left(\mathrm{CH}_{2} \mathrm{O}\right)
\end{aligned}
$$

En faisant la somme des réactions (5) $\times 4$, (6) et (7), nous retrouvons la réaction (2).

\section{La réaction de Hill.}

En 1937, Hill allait faire une découverte qui apportait un argument puissant en faveur de la photolyse de l'eau. Il réussit à obtenir avec des chloroplastes illuminés un dégagement considérable d'oxygène, facilement mesurable quantitativement. Cette production d'oxygène n'avait lieu que si les chloroplastes illuminés se trouvaient en présence d'un oxydant tel que l'oxalate ferrique. L'expérience montrait que l'oxydant était réduit pendant que l'oxygène était émis. Ce dégagement d'oxygène obtenu avec des chloroplastes illuminés, en présence d'un oxydant adéquat, est la manifestation de ce qu'on appelle la réaction de Hill. (Hill, I937 et I939.)

Hill montre qu'il existe une relation stœchiométrique entre la quantité d'oxygène émis et la quantité d'oxydant réduit. Avec l'oxalate de potassium ferrique, tout se passe comme si on avait la réaction (8)

(8)

$$
4 \mathrm{~K}_{3} \mathrm{Fe} \quad\left(\mathrm{C}_{2} \mathrm{O}_{4}\right)_{3}+2 \mathrm{H}_{2} \mathrm{O}+4 \mathrm{~K}^{+} \underset{\text { lumière }}{\stackrel{\text { isolés }}{\text { chloroplastes }}} \rightarrow
$$

$$
4 \mathrm{~K}_{4} \mathrm{Fe}\left(\mathrm{C}_{2} \mathrm{O}_{4}\right)_{3}+4 \mathrm{H}^{+}+\mathrm{O}_{2}
$$

Parmi les accepteurs d'électrons, les plus utilisés dans l'étude de la réaction de Hill, citons le ferricyanure de potassium et la p-benzoquinone introduite par Warburg.
La réaction (8) peut s'écrire sous une forme simplifiée :

(9)

$$
4 \mathrm{Fe}^{+++}+2 \mathrm{H}_{2} \mathrm{O} \underset{\text { lumière }}{\stackrel{\text { chloroplastes }}{\text { isolés }} \rightarrow 4 \mathrm{Fe}^{++}+4 \mathrm{H}^{+}+\mathrm{O}_{2}}
$$

L'oxygène dégagé en fonction du temps peut être mesuré à l'appareil de Warburg. Les autres produits de la réaction $\mathrm{Fe}^{++}$et $\mathrm{H}^{+}$peuvent également être déterminés facilement.

L'examen de l'équation (8) ou (9) montre que $\mathrm{CO}_{2}$ n'intervient pas, ce qui induit Hill à écrire :

"Il est probable que le $\mathrm{CO}_{2}$ ne participe pas à la production d'oxygène. " (Hill, I939.) L'idée que l'oxygène dégagé provient de $\mathrm{H}_{2} \mathrm{O}$, et non pas de $\mathrm{CO}_{2}$, s'impose et l'hypothèse de la photolyse de l'eau vient apporter une interprétation simple de la réaction de Hill :

a) Photolyse de l'eau :

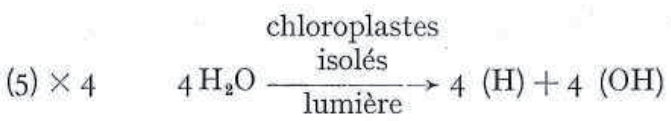

b) Dégagement d'oxygène :

$$
4(\mathrm{OH}) \rightarrow \mathrm{O}_{2}+2 \mathrm{H}_{2} \mathrm{O}
$$

c) Réduction de l'oxydant (ferricyanure de potassium, p. ex.).

La somme des trois réactions précédentes est la réaction de Hill, (9):

(9)

$$
4 \mathrm{Fe}^{+++}+2 \mathrm{H}_{2} \mathrm{O} \underset{\text { lumière }}{\stackrel{\text { chloroplastes }}{\text { isolés }}} \longrightarrow 4 \mathrm{Fe}^{++}+4 \mathrm{H}^{+}+\mathrm{O}_{2}
$$

Nous comprenons maintenant la raison pour laquelle Engelmann, Molish, et tant d'autres, n'obtenaient qu'un dégagement d'oxygène extrêmement faible avec des chloroplastes isolés illuminés : le mélange réactionnel dans lequel étaient placés les chloroplastes isolés ne contenait pas d'oxydant. Le rôle de l'oxydant est capital. Dans l'hypothèse de la photolyse de l'eau, cet oxydant sert à oxyder le système réducteur $(\mathrm{H})$ suivant l'équation (ro) :

$$
4(\mathrm{H})+4 \mathrm{Fe}^{+++} \rightarrow 4 \mathrm{Fe}^{++}+4 \mathrm{H}^{+}
$$


Explicitons cette réaction (I0). Telle que nous l'avons écrite, elle n'indique pas que le système réducteur $(\mathrm{H})$, après avoir été oxydé par $\mathrm{Fe}^{+++}$, est régénéré sous sa forme oxydée. Appelons " $\mathrm{X}$ " cette forme oxydée. On pourra écrire :

$$
(\mathrm{H})=\mathrm{XH} \text {. }
$$

L'équation (Io) devient, en remplaçant $(\mathrm{H})$ par $\mathrm{XH}$ :

$\left(\right.$ Io) ${ }^{\prime} 4 \mathrm{X} \mathrm{H}+4 \mathrm{Fe}^{+++} \rightarrow 4 \mathrm{Fe}^{++}+4 \mathrm{H}^{+}+4 \mathrm{X}$

On voit que $\mathrm{X}$ est régénéré. Le rôle de l'oxydant consiste en fin de compte à régénérer $\mathrm{X}$. Pourquoi est-il nécessaire de le régénérer ? $\mathrm{X}$ est un système enzymatique présent en quantité catalytique seulement. En l'absence d'oxydant, dès les premiers instants où les chloroplastes sont illuminés, tout $\mathrm{X}$ est réduit en $\mathrm{XH}$; la réaction de photolyse de l'eau va s'arrêter, car il n'y aura plus de " $\mathrm{X}$ " disponibles. Pour qu'elle puisse continuer, il convient de régénérer $\mathrm{X}$. C'est le rôle de l'oxydant. La réaction de Hill s'arrêtera quand tout l'oxydant aura été utilisé.

On peut faire le même raisonnement pour $(\mathrm{OH})$ et écrire :

$$
(\mathrm{OH})=\mathrm{YOH}
$$

$(\mathrm{OH})$ ou $\mathrm{YOH}$ est la forme oxydée d'un système enzymatique dont la forme réduite est Y.

L'équation (7) devient :

$$
4 \mathrm{YOH} \rightarrow 2 \mathrm{H}_{2} \mathrm{O}+\hat{\mathrm{O}}_{2}+4 \mathrm{Y} \text {. }
$$

On voit que les $\mathrm{Y}$ sont régénérés; voilà ce qui a lieu chez les plantes vertes. Nous allons voir, au paragraphe suivant, que les bactéries photosynthétiques n'auraient pas justement le pouvoir de régénérer $\mathrm{Y}$ sans secours extérieur.

\section{Le problème posé par les Bactéries Photosyn-} thétiques. L'Équation généralisée de la Photosynthèse.

Alors que le mécanisme précédent expliquait bien les phénomènes photosynthétiques chez les plantes vertes, il demeurerait insuffisant à rendre compte de certaines particularités de la photosynthèse chez les bactéries photosynthétiques.

Il existe trois grands groupes de bactéries photosynthétiques : les Chlorobactéries, les Thiorhodobactéries et les Athiorhodobactéries.

Le tableau I résume les particularités de ces organismes.

\begin{tabular}{|c|c|c|c|c|c|c|}
\hline GROUPE & COULEUR & $\begin{array}{l}\text { BESOIN EN } \\
\text { LUMIÈRE }\end{array}$ & BESOIN EN $\mathrm{O}_{2}$ & $\begin{array}{l}\text { BESOIN DANS LE } \\
\text { MILIEU } \\
\text { DE CULTURE } \\
\text { D'UN COMPOSÉ } \\
\text { RÉDUCTEUR } \\
\text { ORGANIQUE OU } \\
\text { INORGANIQUE }\end{array}$ & $\begin{array}{l}\text { BESOIN EN } \\
\text { VITAMINES }\end{array}$ & RÉSUMÉ \\
\hline $\begin{array}{l}\text { Chloro-bactéries } \\
\text { (ex. Chlorobium) }\end{array}$ & Vert & $\begin{array}{c}\text { Phototrophe } \\
\text { stricte }\end{array}$ & $\begin{array}{l}\text { Anaérobie } \\
\text { stricte }\end{array}$ & Inorganique & Non & $\begin{array}{l}\text { Photoautotrophe } \\
\text { stricte }\end{array}$ \\
\hline $\begin{array}{c}\text { Thiorhodo- } \\
\text { bactéries } \\
\text { (ex. Chromatium) }\end{array}$ & $\begin{array}{c}\text { Pourpre } \\
\text { ou } \\
\text { Rouge }\end{array}$ & $\begin{array}{c}\text { Phototrophe } \\
\text { stricte }\end{array}$ & $\begin{array}{l}\text { Anaérobie } \\
\text { stricte }\end{array}$ & $\begin{array}{l}\text { Inorganique } \\
\text { (autotrophe) } \\
\text { ou Organique } \\
\text { (hétérotrophe) }\end{array}$ & Non & $\begin{array}{c}\text { Photoautotrophe } \\
\text { ou photohétéro- } \\
\text { trophe }\end{array}$ \\
\hline $\begin{array}{l}\text { Athiorhodo- } \\
\text { bactéries } \\
\text { (ex. Rhodospi- } \\
\text { rillum) }\end{array}$ & $\begin{array}{l}\text { Pourpre } \\
\text { Rouge } \\
\text { ou Brun }\end{array}$ & $\begin{array}{c}\text { Phototrophe } \\
\text { ou } \\
\text { Chimiotrophe }\end{array}$ & $\begin{array}{l}\text { Anaérobie } \\
\text { à la lumière } \\
\text { Aérobie à } \\
\text { l'obscurité }\end{array}$ & $\begin{array}{l}\text { Inorganique } \\
\text { ou } \\
\text { Organique }\end{array}$ & $\begin{array}{c}\text { Oui } \\
\text { (hétérotrophe) }\end{array}$ & $\begin{array}{l}\text { Phototrophe } \\
\text { facultatif }\end{array}$ \\
\hline
\end{tabular}

TABLEAU I

Propriétés des Bactéries PhotosynthétiQues. 
Les deux premiers groupes sont des phototrophes stricts : ils ne se développent qu'à la lumière. Les bactéries du troisième groupe sont des phototrophes facultatifs : ils peuvent non seulement se développer à la lumière - et ils se comportent alors comme des anaérobies - mais aussi à l'obscurité et dans ce cas ils ont besoin d'oxygène.

Les bactéries de ces trois groupes sont donc des organismes anaérobies quand ils se développent à la lumière : la photosynthèse a lieu en l'absence d'oxygène. Cette anaérobie stricte oppose les bactéries photosynthétiques aux plantes vertes qui, elles, non seulement se développent en présence d'oxygène, mais encore en dégagent. Tous les essais tendant à montrer un tel dégagement d'oxygène par les bactéries photosynthétiques ont été négatifs.

Une deuxième particularité réside dans le fait que, pour se développer, elles ont besoin, dans leur milieu de culture, d'un composé réducteur. Ce réducteur peut être un composé inorganique, tel que sulfure ou thiosulfate (Chlorobactéries, Thiorhodobactéries) ou un composé organique, tel que l'acétate, le succinate ou le malate p. ex. (Thiorhodobactéries, Athiorhodobactéries).

Cette nécessité d'un réducteur dans le milieu de culture est un caractère remarquable de la physiologie des bactéries photosynthétiques.

Ces bactéries phototrophes ont donc besoin de deux sources d'énergie : la lumière et le réducteur nécessairement présent dans leur milieu de culture. C'est cette double nécessité qui frappe Van Niel : "A mon avis, le problème du métabolisme des bactéries sulfureuses pourpres (Thiorhodobactéries) réside là. Nous devons tenir compte du fait qu'elles ne peuvent vivre qu'en présence de $\mathrm{H}_{2} \mathrm{~S}$ et de lumière. " (Van Niel, I929.) Ces considérations amenèrent alors Van Niel à fairc jouer à ce réducteur le même rôle que l'eau chez les plantes vertes. Aux plantes vertes correspondait la réaction (2).

$$
2 \mathrm{H}_{2} \mathrm{O}+\mathrm{CO}_{2} \rightarrow \mathrm{O}_{2}+\mathrm{H}_{2} \mathrm{O}+\left(\mathrm{CH}_{2} \mathrm{O}\right)
$$

Aux Thiorhodobactéries, avec $\mathrm{H}_{2} \mathrm{~S}$ comme réducteur, correspondra la réaction (II) :

$$
2 \mathrm{H}_{2} \mathrm{~S}+\mathrm{CO}_{2} \rightarrow 2 \mathrm{~S}+\mathrm{H}_{2} \mathrm{O}+\left(\mathrm{CH}_{2} \mathrm{O}\right)
$$

Autrement dit, $\mathrm{H}_{2} \mathrm{~S}$ est considéré comme donneur d'hydrogène et $\mathrm{CO}_{2}$ comme accepteur d'hydrogène. Alors que chez les plantes vertes le produit d'oxydation est l'oxygène, qui se dégage, chez les Thiorhodobactéries, c'est le soufre qui se dépose à l'intérieur des cellules bactériennes. Effectivement, on note souvent un dépôt de soufre à l'intérieur des Thiorhodobactéries. De plus, quand $\mathrm{H}_{2} \mathrm{~S}$ du milieu de culture est épuisé, ces bactéries utilisent le soufre intracellulaire comme réducteur, avec formation d'acide sulfurique comme produit d'oxydation, excrété dans le milieu de culture :

(I2) $2 \mathrm{~S}+5 \mathrm{H}_{2} \mathrm{O}+3 \mathrm{CO}_{2} \rightarrow 3\left(\mathrm{CH}_{2} \mathrm{O}\right)+2 \mathrm{H}_{2} \mathrm{SO}_{4}$

La réaction (I3) est la somme des réactions (II) et ( $\mathrm{r} 2$ ).

(I3)

$2 \mathrm{H}_{2} \mathrm{~S}+4 \mathrm{H}_{2} \mathrm{O}+4 \mathrm{CO}_{2} \rightarrow 4\left(\mathrm{CH}_{2} \mathrm{O}\right)+2 \mathrm{H}_{2} \mathrm{SO}_{4}$

D'après la réaction (I3), il doit donc exister une relation quantitative bien définie, entre $\mathrm{CO}_{2}$ absorbé et $\mathrm{H}_{2} \mathrm{SO}_{4}$ excrété dans le milieu de culture. C'est ce que Van Niel démontre au moyen de toute une série d'expériences. (Van Niel, r929.)

Sûr dé ces résultats, et par analogie avec les équations (2) et (II), Van Niel propose l'équation généralisée suivante :

(I4) $2 \mathrm{H}_{2} \mathrm{~A}+\mathrm{CO}_{2} \rightarrow 2 \mathrm{~A}+\mathrm{H}_{2} \mathrm{O}+\left(\mathrm{CH}_{2} \mathrm{O}\right)$.

Les équations (2) et (II) ne sont alors que des cas particuliers de l'équation (I4). Le cas particulier le plus simple est celui où " $\mathrm{H}_{2} \mathrm{~A}$ ” se résume à $\mathrm{H}_{2}$, l'hydrogène gazeux, et les thiorhodobactéries savent effectivement utiliser l'hydrogène à la place de $\mathrm{SH}_{2}$. " $\mathrm{H}_{2} \mathrm{~A}$ " peut aussi être un composé organique, l'isopropanol par exemple. Dans ce cas, le produit d'oxydation est l'acétone :

$$
\begin{gathered}
\mathrm{CH}_{3} \mathrm{CHOHCH}_{3}+\mathrm{CO}_{2} \rightarrow\left(\mathrm{CH}_{2} \mathrm{O}\right) \\
+\mathrm{H}_{2} \mathrm{O}+2 \mathrm{CH}_{3} \mathrm{COCH}_{3}
\end{gathered}
$$

et l'expérience montre bien que, pour chaque molécule de $\mathrm{CO}_{2}$ fixé, il disparaît 2 molécules d'isopropanol et il apparaît 2 molécules d'acétone. (Van Niel, I94I.)

L'équation généralisée (I4), très largement vérifiée par les nombreuses expériences qu'elle avait suggérées, valable aussi bien avec des réducteurs inorganiques teis que $\mathrm{H}_{2} \mathrm{O}$ (plantes vertes) ou $\mathrm{SH}_{2}$ (Chlorobactéries, Thiorhodobactéries) qu'avec des réducteurs organiques (Rhodobactéries) permettait donc d'expliquer certaines particularités du métabolisme des bactéries photosynthétiques : photosynthèse en l'absence d'oxygène, et nécessité d'un réducteur dans le milieu de culture. Il suffisait de considérer les bactéries comme des organismes qui étaient incapables 
d'utiliser $\mathrm{H}_{2} \mathrm{O}$ comme donneur d'hydrogène alors que les plantes vertes possédaient ce pouvoir. $\left({ }^{*}\right)$

L'équation généralisée était établie sur des bases solides : les relations quantitatives qu'elle imposait entre les produits participant à la réaction se vérifiaient à chaque coup. On tenait là quelque chose de quasi mathématique. Et cependant l'équation généralisée allait être remplacée par une conception plus générale encore. C'est Van Niel lui-même qui allait développer cette nouvelle conception grâce à ses travaux sur le métabolisme des rhodobactéries à l'obscurité.

\section{La Photolyse de 1'eau : dénominateur commun de 1a Photosynthèse.}

Nous avons vu que les Athiorhodobactéries sont des phototrophes facultatifs : en anaérobiose, elles se développent à la lumière ; mais, à condition d'avoir de l'oxygène, elles peuvent aussi se développer à l'obscurité. Van Niel va effectuer de nombreuses expériences pour comparer le métabolisme de ces bactéries à l'obscurité et à la lumière. Voici ce qu'il en conclut :

"Les résultats de nombreuses expériences m'ont convaincu que les représentants aérobies des Athiorhodobactéries peuvent tous être cultivés à l'obscurité dans le même milieu et avec les mêmes substrats **... que ceux utilisés sous des conditions anaérobies à la lumière. " (Van Niel, I94I, p. 304.)

Le même substrat, autrement dit, le même réducteur $\left({ }^{* *}\right)$, peut donc être utilisé pour cultiver ces bactéries, soit à la lumière (en l'absence d'oxygène), soit à l'obscurité (en présence d'oxygène). Van Niel va tirer les conséquences de cette constatation :

"Une culture non illuminée a donc le pouvoir de décomposer le substrat au moyen... de réactions de déshydrogénation qui sont tout à fait indépendantes des processus photochimiques (**)... On est forcé de conclure que la décomposition du substrat est une réaction obscure..."

De plus, aussi bien à l'obscurité en présence d'oxygène qu'à la lumière sous des conditions de stricte anaérobie, la décomposition du substrat procède dans les deux cas avec exactement la même vitesse et elle s'achève après exactement la même durée **. (Van Niel, I94I, p. 304-305.)

(*) Nous verrons dans la deuxième partie de cet article combien cette vue était juste. C'est elle qui, à l'heure actuelle, s'impose à l'esprit.

(**) C'cst nous qui soulignons.
Ainsi donc, chez ces bactéries, la décomposition du substrat, autrement dit la déshydrogénation, du réducteur $\mathrm{H}_{2} \mathrm{~A}$, s'effectue suivant la même réaction, que ce soit à la lumière ou à l'obscurité, et cette réaction, la même dans les deux cas, est une réaction obscure.

Or, d'après l'hypothèse de la photolyse de l'eau, la décomposition de $\mathrm{H}_{2} \mathrm{O}$, le "réducteur spécifique " des plantes vertes, n'est nullement une réaction obscure, mais une réaction photochimique.

On aboutit ainsi à la contradiction suivante : chez les plantes vertes, la décomposition du réducteur est une réaction photochimique; chez les bactéries photosynthétiques, elle est une réaction obscure. Pour tenir compte de cette situation, l'équation généralisée (I4) devrait impliquer deux mécanismes différents : l'un pour les plantes vertes, l'autre pour les bactéries photosynthétiques. Cette solution n'était guère satisfaisante. L'équation généralisée ne représentait plus un concept unifié de la photosynthèsc.

Van Niel va tenter d'apporter une solution élégante au problème. Il écrit : " il n'y a aucune raison de supposer que la réaction photochimique chez les rhodobactéries diffère d'une façon fondamentale de celle qu'on admet avoir lieu chez les plantes vertes. " (Van Niel, I94I, p. 323.)

La réaction photochimique chez les plantes est la photolyse de l'eau :

$$
\left.\mathrm{H}_{2} \mathrm{O} \stackrel{\text { lumière }}{\longrightarrow}(\mathrm{H})+\mathrm{OH}\right)
$$

Chez les bactéries photosynthétiques, nous aurons de même que chez les plantes:

(5) $\times 4 \quad{ }_{4} \mathrm{H}_{2} \mathrm{O} \stackrel{\text { lumière }}{\longrightarrow} 4(\mathrm{H})+4(\mathrm{OH})$

ou, avec les notations définies plus haut:

$$
{ }_{4} \mathrm{H}_{2} \mathrm{O} \stackrel{\text { lumière }}{\longrightarrow} 4 \mathrm{HX}+4 \mathrm{YOH}
$$

Chez les plantes vertes aussi bien que chez les bactéries photosynthétiques (fig. 2 et 3 ), $\mathrm{HX}$ va fournir les hydrogènes nécessaires à la réduction de $\mathrm{CO}_{2}$ :

$$
\text { (I6) }{ }_{4} \mathrm{HX}+\mathrm{CO}_{2} \rightarrow\left(\mathrm{CH}_{2} \mathrm{O}\right)+\mathrm{H}_{2} \mathrm{O}+4 \mathrm{X} \text {. }
$$

Le sort de X H serait le même chez tous les organismes photosynthétiques. C'est essentiellement par le sort de $\mathrm{YOH}$ qui se différencierait la photosynthèse des bactéries photosynthétiques de celle des plantes. 
Chez ces dernières (fig. 2), comme nous l'avons vu, $\mathrm{YOH}$ va donner lieu à un dégagement d'oxygène :

$$
4 \mathrm{YOH} \rightarrow \mathrm{O}_{2}+2 \mathrm{H}_{2} \mathrm{O}+4 \mathrm{Y} \text {. }
$$

Chez les bactéries (fig. 3), un tel dégagement d'oxygène est impossible. $\mathrm{YOH}$ tend à s'accumuler et toutes les réactions photosynthétiques vont s'arrêter quand la totalité des Y sera sous forme de $\mathrm{YOH}$ : cet état est obtenu très rapidement, car $\mathrm{Y}$, système enzymatique, n'est présent qu'en quantité catalytique. Pour que les réactions photosynthétiques puissent continuer, il faut régénérer $Y$, la forme réduite. Van Niel (Van Niel, I94I, p. 324) admet que c'est le réducteur, $\mathrm{H}_{2} \mathrm{~A}$, obligatoirement présent dans le milieu de culture, qui permet la régénération de $\mathrm{Y}$ :

(I8) $4 \mathrm{YOH}+2 \mathrm{H}_{2} \mathrm{~A} \rightarrow 2 \mathrm{~A}+4 \mathrm{H}_{2} \mathrm{O}+4 \mathrm{Y}$

En résumé, chez les plantes vertes (fig. 2):

$$
4 \mathrm{H}_{2} \mathrm{O}+\stackrel{\text { lumière }}{\longrightarrow} 4 \mathrm{HX}+4 \mathrm{YOH}
$$$$
\text { (I6) } 4 \mathrm{HX}+\mathrm{CO}_{2} \rightarrow\left(\mathrm{CH}_{2} \mathrm{O}\right)+\mathrm{H}_{2} \mathrm{O}+4 \mathrm{X}
$$$$
\text { (I7) }+4 \mathrm{YOH} \rightarrow \mathrm{O}_{2}+2 \mathrm{H}_{2} \mathrm{O}+4 \mathrm{Y}
$$$$
\text { (2) } 2 \mathrm{H}_{2} \mathrm{O}+\mathrm{CO}_{2} \stackrel{\text { lumière }}{\longrightarrow} \mathrm{O}_{2}+\mathrm{H}_{2} \mathrm{O}+\left(\mathrm{CH}_{2} \mathrm{O}\right)
$$

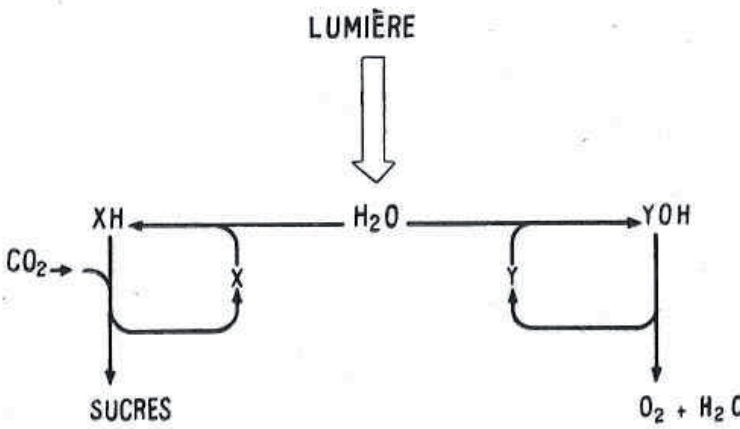

Fig. 2. - Schéma de la photosynthèse chez les plantes vertes suivant les hypothèses de Van Niel (r94r): Photolyse de leau avec génération d'un système réducteur XH, et d'un système oxydant, YOH Élimination des $\mathrm{YOH}$ et "autorégénération » des $\mathrm{Y}$, par dégagement d'oxygène gràce à un système enzymatique qui est l'apanage des plantes vertes.

La somme des réactions $\left(5^{\prime}\right)$, ( $(\mathrm{r} 6)$ et $(\mathrm{I} 7)$ est la réaction (2), cas particulier de l'équation généralisée (I4).

Chez les bactéries photosynthétiques (fig. 3):

$$
4 \mathrm{H}_{2} \mathrm{O} \stackrel{\text { lumière }}{\longrightarrow} 4 \mathrm{HX}+4 \mathrm{YOH}
$$

(I6) $4 \mathrm{HX}+\mathrm{CO}_{2} \rightarrow\left(\mathrm{CH}_{2} \mathrm{O}\right)+\mathrm{H}_{2} \mathrm{O}+4 \mathrm{X}$

(I8) $+4 \mathrm{YOH}+2 \mathrm{H}_{2} \mathrm{~A} \rightarrow 2 \mathrm{~A}+4 \mathrm{H}_{2} \mathrm{O}+4 \mathrm{Y}$

(I4) $2 \mathrm{H}_{2} \mathrm{~A}+\mathrm{CO}_{2} \stackrel{\text { lumière }}{\longrightarrow} 2 \mathrm{~A}+\mathrm{H}_{2} \mathrm{O}+\left(\mathrm{CH}_{2} \mathrm{O}\right)$

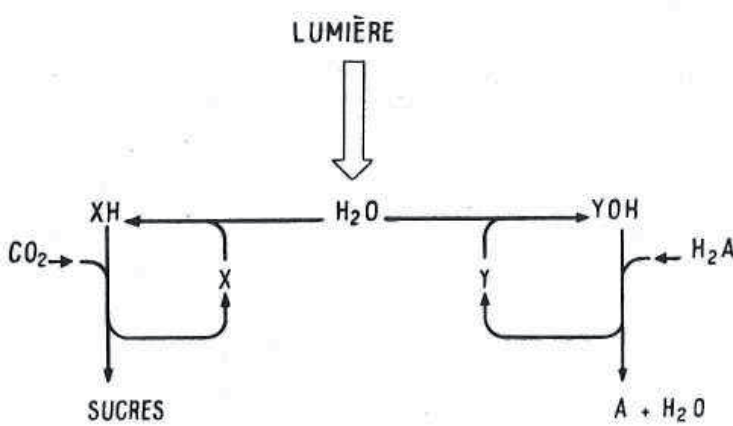

FIG. 3. - Schéma de la photosynthèse chez les bactéries photosynthétiques suivant les hypothèses de Van Niel (r94I):

Photolyse de l'eau avec génération d'un système réducteur, $\mathrm{XH}$, et d'un système oxydant, YOH. Élimination des YOH par réaction ave un réducteur $\mathrm{H}_{2} \mathrm{~A}$; $\mathrm{Y}$ est ainsi régénéré, et la forme oxydéc, $\mathrm{A}$, du réducteur, $\mathrm{H}_{2} \mathrm{~A}$, s'accumule.

La somme des réactions ( $5^{\prime}$ ), (I6) et (I8) est la réaction (I4) ou équation généralisée, que nous retrouvons ici.

Quelle est la nouveauté des conceptions de Van Niel ?

Dans le concept de l'équation généralisée, $\mathrm{H}_{2} \mathrm{O}$ pour les plantes vertes, et " $\mathrm{H}_{2} \mathrm{~A}$ " pour les bactéries photosynthétiques, jouaient le même rôle. Par analogie avec le rôle de $\mathrm{H}_{2} \mathrm{O}$ chez les plantes, " $\mathrm{H}_{2} \mathrm{~A}$ " était considéré comme un donneur d'hydrogène servant à réduire $\mathrm{CO}_{2}$. Le dénominateur commun de la photosynthèse chez les plantes et chez les bactéries était la nécessité d'un réducteur " $\mathrm{H}_{2} \mathrm{~A}$ " qui, chez les plantes, ne pouvait être que $\mathrm{H}_{2} \mathrm{O}$ et qui, chez les bactéries, pouvait être un corps inorganique tel que $\mathrm{H}_{2} \mathrm{~S}$, ou un composé organique tel que $\mathrm{CH}_{3}-\mathrm{CHOH}-\mathrm{CH}_{3}$, mais pas $\mathrm{H}_{2} \mathrm{O}$.

Dans la nouvelle conception de Van Niel, le dénominateur commun de la photosynthèse devient la photolyse de l'eau. Chez les bactéries photosynthétiques, aussi bien que chez les plantes vertes, l'énergie lumineuse sert à réaliser une décomposition de l'eau en un système réducteur $(\mathrm{H})$ et un système oxydant $(\mathrm{OH})$. Dans tous les cas, c'est $(\mathrm{H})$ qui sert à réduire $\mathrm{CO}_{2}$ : autrement dit, dans tous les cas c'est $\mathrm{H}_{2} \mathrm{O}$ qui est la source première de l'hydrogène nécessaire à la réduction de $\mathrm{CO}_{2}$. Le seul rôle du réducteur obligatoirement présent dans le milieu de culture anaérobie 
des bactéries photosynthétiques, consiste à éliminer $(\mathrm{OH})$ ou $(\mathrm{YOH})$; autrement dit, à réduire la forme oxydée $\mathrm{YOH}$ d'un système enzymatique qui, ainsi régénéré sous sa forme réduite $\mathrm{Y}$, peut à nouveau entrer en réaction.

Les plantes vertes n'ont pas besoin d'un tel réducteur, car elles éliminent les $(\mathrm{OH})$ en dégageant de l'oxygène, ce qui régénère $\mathrm{Y}$ automatiquement.

Nous avons vu, un peu plus haut, que la somme des réactions $\left(5^{\prime}\right),(16)$ et $(\mathrm{I} 8)$ donnait l'équation généralisée (I4). En effet, les nouvelles hypothèses de Van Niel ne contredisent pas l'équation généralisée, à condition de considérer cette équation comme la simple expression d'une relation quantitative entre $\mathrm{CO}_{2}$ réduit et $\mathrm{H}_{2} \mathrm{~A}$ oxydé et non pas comme un mécanisme : “ ... on doit bien réaliser que l'équation généralisée de la photosynthèse indique simplement que la réduction du gaz carbonique dépend de la déshydrogénation du composé $\mathrm{H}_{2} \mathrm{~A}$; elle n'est pas l'expression d'un mécanisme». (Van Niel, I94I, p. 323.)

Le nouveau schéma de la photosynthèse développé par Van Niel impliquait davantage qu'une simple dépendance entre produits d'une réaction. Il représentait un mécanisme de la photosynthèse. Il fournissait surtout une hypothèse de travail simple. Van Niel terminait par les lignes suivantes son article de I94I sur la photosynthèse : "En tant qu'hypothèse de travail simple... [le nouveau schéma]... présente certains avantages; on ne pourra juger de sa valeur que par les travaux futurs qui en découleront. ”

Cette hypothèse de travail a été extrêmement fructueuse pendant les 20 dernières années.

Par les travaux qu'elle a stimulés, elle a fait progresser d'une façon considérable l'étude de la photosynthèse. Cependant à la lueur des nouvelles conceptions développées par D. I. Arnon et F. R. Whatley, et leurs collaborateurs à l'Université de Callfornie, à Berkeley, il semble que l'hypothèse de Van Niel d'après laquelle la photolyse de l'eau est le dénominateur commun de la photosynthèse, soit fausse pour des raisons que nous exposerons au cours de la deuxième partie de cet article.

\section{Les expériences de Photosynthèse avec $\mathrm{H}_{2} \mathrm{O}^{18}$.}

Nous venons de voir comment l'étude comparée de la photosynthèse chez les plantes et chez les bactéries photosynthétiques ont conduit Van Niel à voir dans la photolyse de l'eau le dénominateur commun de la photosynthèse et comment la réaction de Hill a pu être considérée comme une confirmation expérimentale de la photolyse chez les plantes vertes.

D'après ces théories et ces expériences, l'oxygène dégagé pendant la photosynthèse devait provenir de l'eau. C'est ce phénomène que démontrèrent expérimentalement Ruben et coll. d'une part, Holt et French d'autre part.

Ruben et coll. (Ruben, I94I) firent photosynthétiser des cellules de Chlorella, algue verte monocellulaire, en présence d'eau enrichie en oxygène $I 8$, isotope lourd de l'oxygène "ordinaire" de nombre de masse 16.

Le pourcentage d'oxygène 18 dans l'eau était de 0,85 . Ils trouvèrent que le pourcentage d'oxygène I8 dans l'oxygène dégagé par les algues éclairées était aussi de 0,85 . L'identité de ces pourcentages les amenait à conclure que l'eau était la source de l'oxygène dégagé pendant la photosynthèse.

Quelques années plus tard, Holt et French (Holt, I948) firent le même type d'expérience, mais avec des chloroplastes isolés d'épinards au lieu de cellules entières d'algues. Autrement dit, ils effectuèrent une réaction de Hill en présence d'eau enrichie en oxygène I8. Ils trouvèrent que, quel que soit l'oxydant utilisé pour la réaction de Hill, la composition en $\mathrm{O}^{18}$ de l'oxygène dégagé photosynthétiquement correspondait exactement à celle de l'oxygène produit par électrolyse de la même eau (même pourcentage de $\mathrm{O}^{18}$ ). Cela prouvait bien que, dans la réaction de Hill, l'oxygène provenait de $\mathrm{H}_{2} \mathrm{O}$, quel que fut l'«oxydant " :

$$
2 \mathrm{H}_{2} \mathrm{O}^{18}+\mathrm{Fe}^{+++} \frac{\text { lumière }}{\substack{\text { chloroplastes } \\ \text { isolés }}} \mathrm{O}_{2}{ }^{18}+4 \mathrm{H}^{+}+\mathrm{Fe}^{++}
$$

Cette expérience représente aussi une élégante illustration de l'analogie entre le dégagement d'oxygène pendant la réaction de Hill et le dégagement d'oxygène qui se produit à l'anode d'une cuve à électrolyse (voir précédemment).

\section{Réactions " claires " (photochimiques) et réactions "sombres" (non photochimiques).}

Nous avons déjà été amené, à parler, au cours des pages précédentes, de réactions claires, ou photochimiques et de réactions sombres ou non photochimiques. Ainsi, dans le schéma de la photosynthèse de Van Niel, la photolyse de l'eau est une réaction photochimique alors que, par exemple, la déshydrogénation du réducteur nécessaire aux rhodobactéries est une réaction sombre.

La notion d'après laquelle les réactions photosynthétiques sont composées de réactions photochi- 
miques et de réactions non photochimiques est antérieure à la théorie de Van Niel. Elle est due, en particulier, à Blackmann (Ig05) et à Warburg (I928). Elle était basée, entre autres, sur le fait qu'une certaine quantité de lumière de forte intensité est capable de "réduire" davantage de $\mathrm{CO}_{2}$ lorsqu'on la fournit sous forme d'une succession d'éclairs de courte durée, que sous forme de lumière continue. (Warburg, I928.) L'existence de réactions claires et de réactions sombres permettait d'expliquer cette augmentation : pendant la période d'illumination, les produits des réactions photochimiques s'accumulent et pendant la période sombre les réactions non photochimiques ont le temps d'agir sur ces " photoproduits" accumulés. L'utilisation de la lumière est d'autant meilleure que les réactions sombres sont plus complètes.

Dans le schéma de Van Niel, la réaction photochimique est la photolyse de l'eau qui se traduit par l'apparition de $(\mathrm{H})$ et de $(\mathrm{OH})$. L'utilisation de $(\mathrm{H})$ et de $(\mathrm{OH})$ est le résultat de réactions sombres. En particulier, la réduction du $\mathrm{CO}_{2}$ (ou d'un produit de carboxylation) apparaît comme une réaction (ou une série de réactions) sombre.

L'idée que des réactions sombres fussent responsables de la fixation et de la réduction de $\mathrm{CO}_{2}$. s'est établie fermement à partir de 1936 , quand on a commencé à mettre en évidence que des organismes, non photosynthétiques, avaient le pouvoir de fixer et de réduire $\mathrm{CO}_{2}$. Van Niel pouvait écrire, en I94I : "Le pouvoir de certains organismes de réduire le gaz carbonique, sans apport d'énergie radiante, est un phénomène très général ». (Van Niel, I94I, p. 322.) Nous verrons, dans la deuxième partie de cet article, comment on est effectivement arrivé, en I958, à séparer, dans le temps et dans l'espace, les réactions photochimiques des réactions sombres.

\section{Le rôle du chloroplaste tel qu'il apparaît à la suite des travaux précédents.}

La découverte de la réaction de Hill montrait excellemment que les chloroplastes étaient le siège de la photolyse de l'eau, de la réaction photochimique. Que les chloroplastes fussent le siège des réactions photochimiques était assez évident, puisque là étaient localisés, entre autres, les pigments chlorophylliens qui permettaient l'absorption de l'énergie lumineuse. Mais les chloroplastes étaient-ils aussi le siège de la fixation et de la réduction de $\mathrm{CO}_{2}$ ? Alors, mais alors seulement, les chloroplastes eussent bien été le siège de toute la photosynthèse.

Dans les années I940, tous les essais tendant à mettre en évidence une absorption de $\mathrm{CO}_{2}$ par des chloroplastes isolés ont échoué, même ceux où fut utilisé du gaz carbonique marqué au Carbone $\mathrm{I}_{4}, \mathrm{CO}_{2}{ }^{14}$.

Aussi, à partir de 1950 , était-il couramment admis que le chloroplaste était un "système beaucoup plus simple que celui nécessaire pour la photosynthèse " (Lumry, I954) et qu'il était uniquement le lieu où la lumière était absorbée, et le siège des réactions de photolyse de l'eau.

Nous allons voir, au cours de la deuxième partie, qu'il n'en est rien et que le chloroplaste est véritablement le siège de toutes les réactions photochimiques.

(A suivre.)

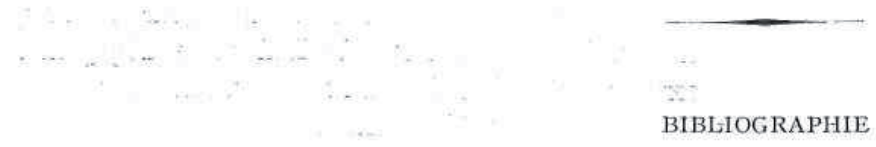

Audubert (R.), 1953. - Electrolyse, Presses Universitaires de France.

BUChNer (E.), I987. - Ber. Deut. Chem. Ges., 30, II7-124.

DUtrochet (R. J. H.), I837. - Mémoire pour servir à l'histoire anatomique et physiologique des végétaux et des animaux. Bruxelles,

Engelmann (Th. W.), 1882, - Ueber Sauerstoffausscheidung von Pflanzenzellen im Mikrospectrum. Botanische Zeitung, 4o, $419-426$.

HANSEN (A.)., 1882. - Geschichte der Assimilation und Chlorophyll-function. In : Arbeiten des botanischen Institut in Würzburg. Heft $4,537-626$.

Hill (R.), 1937. - Nature, $139,88 \mathrm{r}$.

HrL. (R.), r 939. - Proceedings of the Royal Society of London, B. $127,192-210$.

Holt (A. S.) and Frencir (C. S.), 1948. - Arch. Biochem, 19, 429435 .
INGEN-Housz (J.), I779. - Experiments upon vegetables, discovering their great power of purifying the common air in the sunshine and of injuring it in the shade and at night. London.

Lumry (R.), Spikes (J. D.) and Eyring (H.), I954. - Ann. Rev. Plant Physiol., 5, $27 \mathrm{x}$.

MAYER (J. R.), I845. - Die organische Bewegung in ihrem Zusammenhange init dem Stoffwechsel. Heilbronn.

Мoнl (H. v.), I837. - Untersuchungen über die anatomischen Verhältnisse des Chlorophylls. Univ. Tübingen.

Molish (H.), I925. - Z. Botan., $17,578$.

Prerfer (W.), rgoo, - The Physiology of plants. Clarendon, Oxford, vol. I.

Presstley (J.), 1772. - Philosophical Transactions of the Royal Society of London, 62, 166-170.

Priestley (J.), I776. - Experiments and Observations on different kinds of air, London. 
Rubex (S.), Randall (M.), KaMen (M.) and Hyde (J. L.), 1941. Journal of the American Chemical Society, $6.3,877-879$.

SACHS (J.), 1887. - Lectures on the physiology of plants. Clarenden, Oxford.

SAussure (N. T, DE), I804. - Recherches chimiques sur la végétation. Paris.

Sexebier (J.), r782, - Mémoires physico-chimiques sur l'influence de la lumière solaire pour modifier les être des trois règnes de la nature, et sur tous ceux du règne végétal. Genève.

Senebier (J.), I873. - Recherches sur l'influence de la lumic̀re solaire pour métamorphoser l'Air fixe en Air pur par végétation.

VAN NiEL (C. B.), 1929. - Contributions to Marine Biology (A report of the 1929 Meeting of the Western Society of Naturalists) Stanford University Press, I6r-169.

VAN Nrfu (C. B.), I94I. - The bacterial photosynthesis and their importance for the general problem of photosynthesis. Ad. vances in Enzymology, $x, 263-328$.

WARBURG (O.), 1928. - Ueber die katalytischen Wirkungen der lebendigen Substanz. Julius Springer-Verlag.

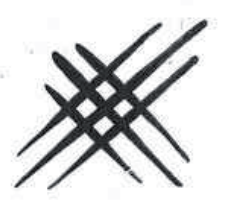

\section{POUR LE DESHERBAGE DE LA CANNE A SUCRE FLY-TOX \\ vous propose}

outre les WEEDONE lesters lourds à base de 2-4 D) et I'HERBOXY-S (à base de Simazine)

\section{le GES A P IME (50\% d'Atrazine)}

- Sélectivité parfaite.

- Efficacité herbicide très étendue.

- Très grande rémanence.

- Doses d'emploi: 4 à $5 \mathrm{~kg} /$ hectare.

- Peut également être utilisé sur Ananas, Bananes, etc...

Société LE FLY-TOX - Service exportation, B. P. 5I - GENNEVILLIERS (Seine). 